\title{
Injection Mold Cavity Stiffness and Intensity Analysis Based on ANSYS Workbench and MOLDFLOW
}

\author{
GENG Tie $^{1, a}$, TU Wei-qing ${ }^{1, b}$, LIU Dan-dan ${ }^{1, c}$ \\ ${ }^{1}$ Collage of Mechanical \& Electrical Engineering, Henan University of Technology, Zhengzhou \\ 450007, China \\ atiegeng2000@163.com, bweiqing00515@163.com, cliudan5251@126.com
}

Keywords: Stiffness and intensity; ANSYS Workbench; MOLDFLOW; Finite element

\begin{abstract}
The loading conditions of cavity is determined through using CAE software MOLDFLOW to simulate plastic flow injection molding process of the plastic product and as the force for the input load of the plastic products injection mold cavity strength analysis. Study on finite element simulation of stress and strain of the cavity using ANSYS Workbench. The results of the study show that the integrated using software ANSYS Workbench and MOLDFLOW can comprehensively reflect the cavity's force and distortion situation in injection molding process, thus providing more valuable reference for mold design.
\end{abstract}

\section{Introduction}

In injection molding, the cavity must have sufficient stiffness and intensity to bear pressure of high-temperature melt. If the cavity stiffness is insufficient, its elastic deformation will take place, thus causing larger gap in contact or mating surface of the molding parts and burrs or flying edge, reducing the accuracy of the plastics and impacting demolding.If the strength is insufficient, which will bring about plastic deformation even cracking ${ }^{[1-2]}$.

When checking cavity stiffness and strength, the traditional methods relied principally on the experience and simple calculation formula. Although the design method can guarantee the mould stiffness, it is easy to waste the mold material. In recent years, CAE technology is developing rapidly, used in various fields. In the field of injection mould, through using the CAE technology a lot of research results have been produced ${ }^{[3-4]}$. However, these studies mainly focus on the simulation of injection molding process and parameters optimization. According to the problems above, this article takes an injection mold cavity as the research object and using the CAE software MOLDFLOW ${ }^{[5]}$ and ANSYS Workbench ${ }^{[6]}$ to simulate the plastic parts forming process and mold force and deformation of cavity. The injection mold cavity is shown in Fig.1.

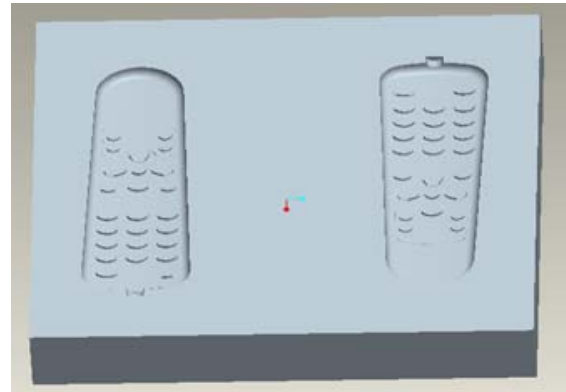

Fig.1 Injection mold cavity $3 \mathrm{~d}$ model

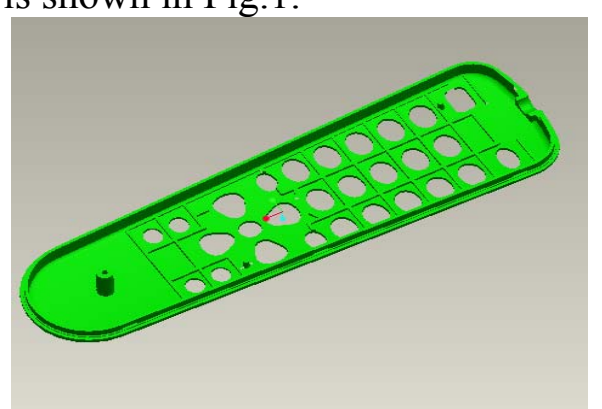

Fig.2 Remote control front cover 3d model

\section{The theoretical basis of stiffness and intensity analysis ${ }^{[7]}$}

The finite element method is one of the important techniques in analysis of engineering problems. This method has been successfully applied in the fields of elastic mechanics、 dynamics problems、 elastoplasticity v contact mechanics 、creep 、 fatigue and fracture mechanics, fluid mechanics, thermodynamics and so on. The theoretical basis of the injection mold cavity stiffness and intensity analysis is finite element method, which discreteness geometry into a finite number of elements, 
makes simultaneous solution of each element body by applying basic equations as well as the principle of minimum potential energy, and comes to meet the engineering precision approximation results to alternate the analysis of the actual objects. As follows is the process of using the finite element method for stress and strain analysis.

For a continuous elastic body, after being discredited in the finite element method, the elements are passed load through nodes, the displacement function $U$ of the elements can be expressed as

$$
U=N u^{e}
$$

Where, $U$ is the displacement vector of any element, $N$ is the shape function matrix; $U^{e}$ is the displacement vector of any element node.

According to the geometric equations of continuous elastomeric and formula (1), and to make $B=L N$, then the strain vector $\varepsilon$ of any element can be expressed as

$$
\varepsilon=L U=L N u^{e}=B u^{e}
$$

Where, $\varepsilon$ is the strain vector of any element, $L$ is the differential operator; $B$ is the element strain matrix.

According to the physical equations of continuous elastomeric and formula (2), the stress vector $\sigma$ of any element can be expressed as

$$
\sigma=D \varepsilon=D B u^{e}
$$

Where, $\sigma$ is the stress vector of any element, $D$ is the elastic matrix.

For any element, the stiffness equation of element can be organized by bringing the displacement formula (1), strain formula (2) and stress formula (3) into the principle of virtual work

$$
K^{e} U^{e}=P^{e}
$$

Where, $K^{e}$ is the stiffness matrix of elements, $P^{e}$ is the equivalent nodal load vector of element.

Finally, assume that the object is discredited into $n_{e}$ elements; the overall stiffness equation of the geometry can be gotten by Adding up all $n_{e}$ stiffness equation of elements

$$
K U=P
$$

\section{Founded the finite model}

Lead cavity 3D geometric model into finite element analysis software ANSYS Workbench, and select material, modulus of elasticity and Poisson ratio are $2.2 \times 10^{5} \mathrm{MPa}$ and 0.25 . The Hex Dominant mesh method (the combination way of tetrahedron and hexahedron) was used. Because the cavity can't be simplified in some structure, refine the grid to improve the analysis accuracy. The number of grid is 16193 , node is 28631 . The injection mold cavity finite element model was shown in Fig.3.

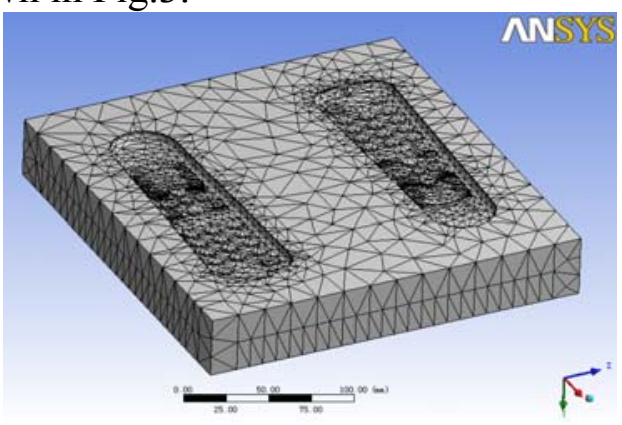

Fig.3 Finite element model of cavity

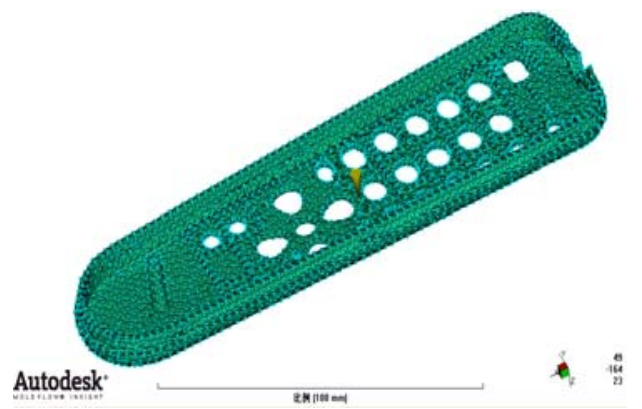

Fig.4 Finite element model of front cover

\section{Dispose of boundary conditions}

\section{Load}

In the process of injection molding, the pressure mold cavity suffered is as same as the injection pressure thin-walled plastic products suffered. With the thickness $1.5 \mathrm{~mm}$, and length $174 \mathrm{~mm}$, Remote control front cover belongs to the thin-walled plastic products, as shown in Fig. 2. 
Finite element modeling. Convert geometry models of the remote controller' front cover into STL format, then import in MOLDFLOW, using a two-level grid model for meshing, diagnosed by grid, auto repair. The repaired finite element model is shown in Fig.4.The number of grid is 11432 and node 7448 .

Mold flow analysis results. The main purpose of the mold flow analysis is to extract the maximum pressure in the cavity, and provides input loads to the analysis of stiffness. As shown in the Fig.5, the injection molding process places the injection pressure changing with time curve. The Fig.5 shows when $\mathrm{t}$ is $0.3301 \mathrm{~s}$, the pressure is greatest and the value is $30.05 \mathrm{Mpa}$.

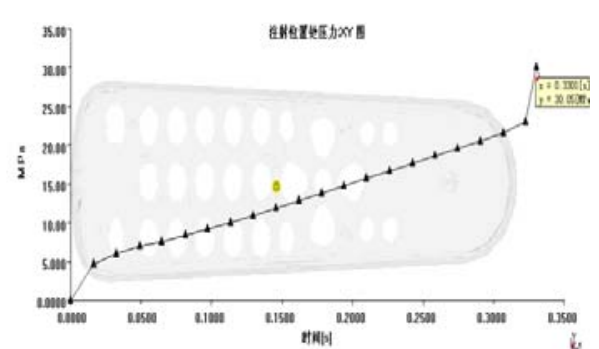

Fig.5 Pressure at injection location

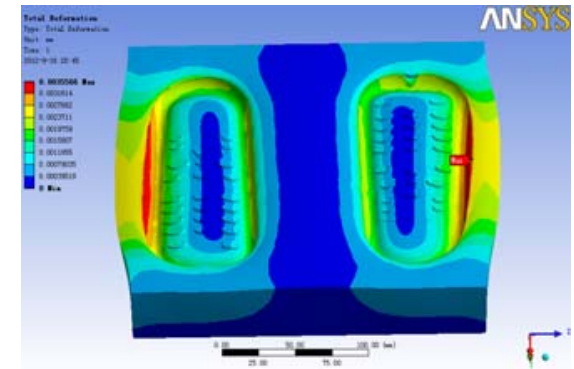

Fig.7 The equivalent stress of cavity

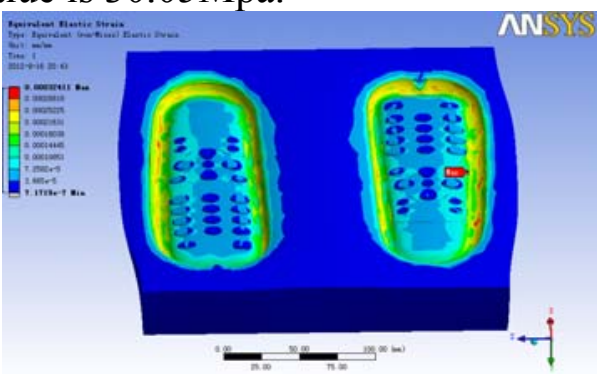

Fig.6 The deformation of cavity

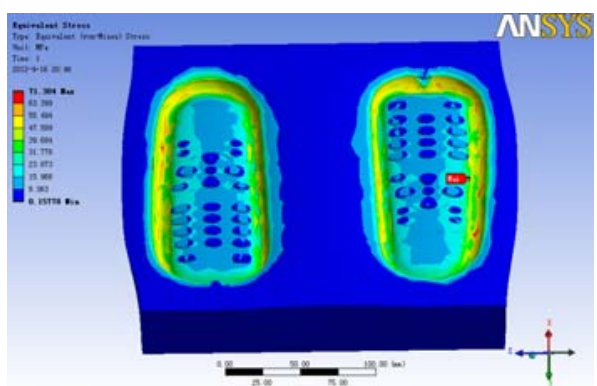

Fig.8 The equivalent strain of cavity

\section{Restriction}

The cavity is monolithic cavity, so the cavity bottom is applied fixed constraint, limiting its six degree of freedom.

\section{Results and analysis}

As shown in Fig.6, the deformation of the wall by the center to reduce gradually, so central wall thickness is very important and should be considered in the design.

Cavity stiffness calculation standards require that the maximum elastic deformation cavity should not be more than plastic product size tolerance one forth to one fifth and parting the maximum clearance, to ensure the accuracy of plastic products. For the remote controller's front cover who use the ABS material, its deformation should be controlled in 0.05 to $0.08 \mathrm{~mm}$ below. As shown in Fig.7, the cavity of the maximum deformation is $0.00355 \mathrm{~mm}$ whose value is far less than the allowable deformation. So this cavity stiffness meets the requirements.

Mold strength calculation standards cavity by external force stress value should not exceed allowable stress, that is $\sigma<[\sigma]$. As shown in Fig.8, the maximum stress is $71.304 \mathrm{Mpa}$, material allowable stress is $300 \mathrm{MPa}$. So the mold strength is enough.

The analysis results show that the cavity can meet the requirements of the stiffness and strength. But the size design is too conservative, wasting material. Therefore, based on the analysis results optimization design can be carried out for the cavity in future, which can reduce the mold production cost.

\section{Conclusion}

Research shows that the integrated using software ANSYS Workbench and MOLDFLOW as injection molds stiffness and strength analysis tools, which can comprehensively reflect the cavity' s force and distortion situation in injection molding process, thus providing scientific and reliable 
theoretical foundation for mold design and condition for subsequent development of injection mold stiffness and strength analysis system.

\section{References}

[1] Li Qi-xin.The points of rigidity calculation for injection mold.Electromechanical Component, 1997, 14 (12): $: 55-60$

[2] Li Yun.Intensity and stiffness Analysis for Plastic Mould Thickness. Mould Design, 2008(12): $78-80$

[3] J.Koszkul, J.Nabialek.Viscosity models in simulation of the filling stage of the injection molding process [J].Journal of Materials Processing Technology, 157-158(2004):183-187

[4] Yuan Zhong-shuang. The progress and trend of injection mold CAE technology. China Mechanical Engineering, 1992.3(6):13-16

[5] Chen Yan-xia, Chen Ru-xiang.MOLDFLOW2010 complete self-study and fast manual [M]. Beijing: Publishing House of Electronics Industry

[6] Li Bing, He Zheng-jia.ANSYS Workbench design simulation and optimization [M]. Beijing: TsingHua University Press

[7] Wang Xu-cheng.The Finite element method [M].Beijing: TsingHua University Press 